\title{
The Mean-Variance-CVaR model for Portfolio Optimization Modeling using a Multi-Objective Approach Based on a Hybrid Method
}

\author{
R. Aboulaich , R. Ellaia and S. El Moumen* \\ LERMA, EMI, Av. Ibn Sina B.P. 765 Agdal, Rabat, Morocco
}

\begin{abstract}
In this paper we present a new hybrid method, called SASP method. We propose the hybridization of two methods, the simulated annealing (SA), which belong to the class of global optimization based on the principles of thermodynamics, and the descent method were we estimate the gradient using the simultaneous perturbation. This hybrid method gives better results. We use the Normal Boundary Intersection approach (NBI) based on the SASP method to solve a portfolio optimization problem. Such problem is a multi-objective optimization problem, in order to solve this problem we use three statistical quantities: the expected value, the variance and the Conditional Value-at-Risk (CVaR). The purpose of this work is to find the efficient boundary of the considered multi-objective problem using the NBI method based on the SASP method.
\end{abstract}

Key words: CVaR, multi-objective optimization, NBI method, hybrid method SASP, simultaneous perturbation, simulated annealing

AMS subject classification: 46N10, 74P10

\section{Introduction}

Mean-risk models are still the most widely used approach in the practice of portfolio selection. With mean-risk models, return distributions are characterized and compared using two statistical quantities: the expected value and the value of a risk measure. Thus, mean-risk models have a ready interpretation of results and in most cases are convenient from a computational point of view. On the other hand in the practice of describing a distribution by just two parameters involves great loss of information. It is well known that the risk measure plays an important role in making the decisions. Variance was the first risk measure used in mean-risk models (Markowitz 1952 [5])

${ }^{*}$ Corresponding author. E-mail: selmoumen@yahoo.fr 
and, in many proposals of new risk measures. Another risk measure, the Conditional Value-atRisk (CVaR) ([1], [2], [7])), is growing in popularity. CVaR has attractive theoretical properties: it controls the magnitude of losses beyond VaR and it is coherent. In this paper we propose to solve the problem of portfolio selection, by using the NBI [3] approach based on a new hybrid algorithm SASP, implemented in Matlab.

\section{The Mean-Variance-CVaR model}

Now a model for portfolio selection is proposed, in which random variables are described by three statistical quantities: the expected value, the variance and the CVaR. We claim that taking three parameters into consideration, instead of two, gives a better modelling power. The proposed model may bring an improvement in the solution, in the case where a mean-variance efficient portfolio has an excessively large CVaR, or a mean-CVaR efficient portfolio has an excessively large variance. In the mean-variance-CVaR model, a random variable $R_{x}$ is preferred to a random variable $R_{y}$ if: $E\left(R_{x}\right) \geq E\left(R_{y}\right), \sigma^{2}\left(R_{x}\right) \leq \sigma^{2}\left(R_{y}\right)$ and $C V a R\left(R_{x}\right) \leq C V a R\left(R_{y}\right)$. The Mean-Variance-CVaR model can be formulated as follows:

$$
(P)\left\{\begin{array}{l}
\text { Minimize }\{C V a R,-E, \text { var }\} \\
\text { Subject to }: x \in\left\{\left(x_{1}, \ldots, x_{n}\right) / \sum_{i=1}^{n} x_{j}=1, x_{j} \geq 0, \forall j \in\{1, \ldots, n\}\right\} .
\end{array}\right.
$$

To solve this problem we use the NBI approach based on a hybrid method SASP.

\section{The SASP method}

The SASP method is used to find the global optimum. The proposed method (SASP) is obtained by hybridization of the two methods, the simulated annealing algorithm [4] and the descent method where the gradient [6] is estimate by using the simultaneous perturbation.

First of all, we use the gradient method to find a local solution, to escape from this local solution we use some iterations of simulated annealing algorithm to find the first better point, then we take this better point as an initial point and begin a new research with the gradient method. This procedure goes on until convergence and finally we find the global solution, see Figure 1.

\section{Numerical Example}

\subsection{Benchmark functions}

To illustrate the efficiency of the proposed Hybrid method SASP, we propose to solve some test functions. These test functions have different features like convex/nonconvex, continuous, unimodal/multi-modal etc, with known global optima, with multiple local minima. Finally, our proposed technique have been compared with some existing methods and the comparative study is 


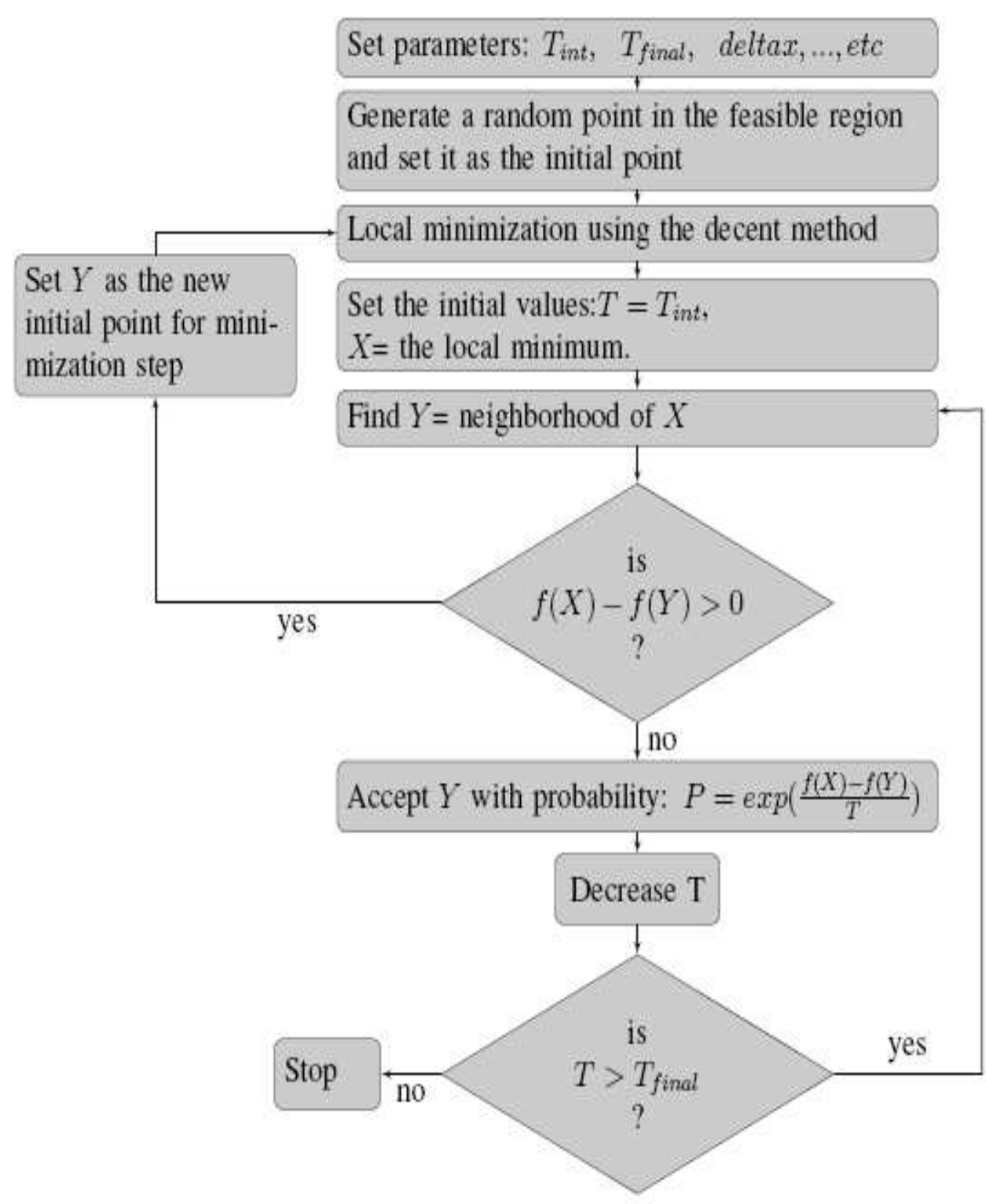

Figure 1: Flow chart of the SASP algorithm.

displayed in the following Table 1. The results of SASP listed in Table 1 compared with results of other methods, indicates that the results of SASP are very encouraging, reliable and efficient. 


\begin{tabular}{ccccc}
\hline \multirow{2}{*}{ Test function } & \multicolumn{2}{c}{ SASP method } & \multicolumn{2}{c}{ Other Methods } \\
\cline { 2 - 5 } & Dimension & Obtained result & Method & Obtained result \\
\hline Rastrigin's function & 10 & $2.1316 \mathrm{e}-014$ & HGA [8] & $1.29 \mathrm{e}-12$ \\
& & & GAT [8] & 1.37 \\
& 50 & $1.4531 \mathrm{e}-012$ & HGA & $9.09 \mathrm{e}-12$ \\
& 100 & $1.3975 \mathrm{e}-011$ & GAT & 143.37 \\
& & & GAT & $2 \mathrm{e}-11$ \\
AlluffiPentiny's function & 2 & -0.352386 & MGA [9] & -0.352386 \\
Bohachevsky's function & 2 & 0 & MGA & 0 \\
\hline
\end{tabular}

Table 1: Computational results of test functions.

\subsection{Portfolio example}

Let (ONA, GAZ, SAMIR, ATWB) be the assets, quoted in the Casablanca Stock Exchange, with history of 2 years(01/2007 - 12/2008). We use the NBI method based on the hybrid method to find the efficient frontier of the mean-variance-CVaR model $(P)$. The results are provided by Figure 2. As we can see, from Figure 2, it is clearly that the NBI, based on the hybrid method, parametrization of the efficient frontier naturally bears the property of even spread. Also the figure can be shown that our approach guarantees uniform distribution and exclude the non-Pareto and local Pareto points.

\section{Conclusion}

A new hybrid method is proposed in this paper, called SASP method. The hybridization of two methods, the simulated annealing, and the descent method were we estimate the gradient using the simultaneous perturbation. We have tested the proposed algorithm with other algorithms using some Benchmark functions, and the numerical results confirm that our SASP has powerful ability to find global optimums. In this work, we proposed also the Normal Boundary Intersection approach (NBI) based on the SASP method to solve a portfolio optimization problem. The problem of selecting an efficient solution of these models is multi-objective. We chose CVaR because it measures the average loss over a specified number of worst cases.

\section{References}

[1] R. T. Rockafeller, S. Uryasev. Optimization of conditional value-at-risk. Journal of Risk, 2 (2000), No. 3 , 21-42.

[2] S. Uryasev. Conditional value-at-risk: optimization algorithms and applications. Financial Engineering News, (2000), No. 14, 1-5. 

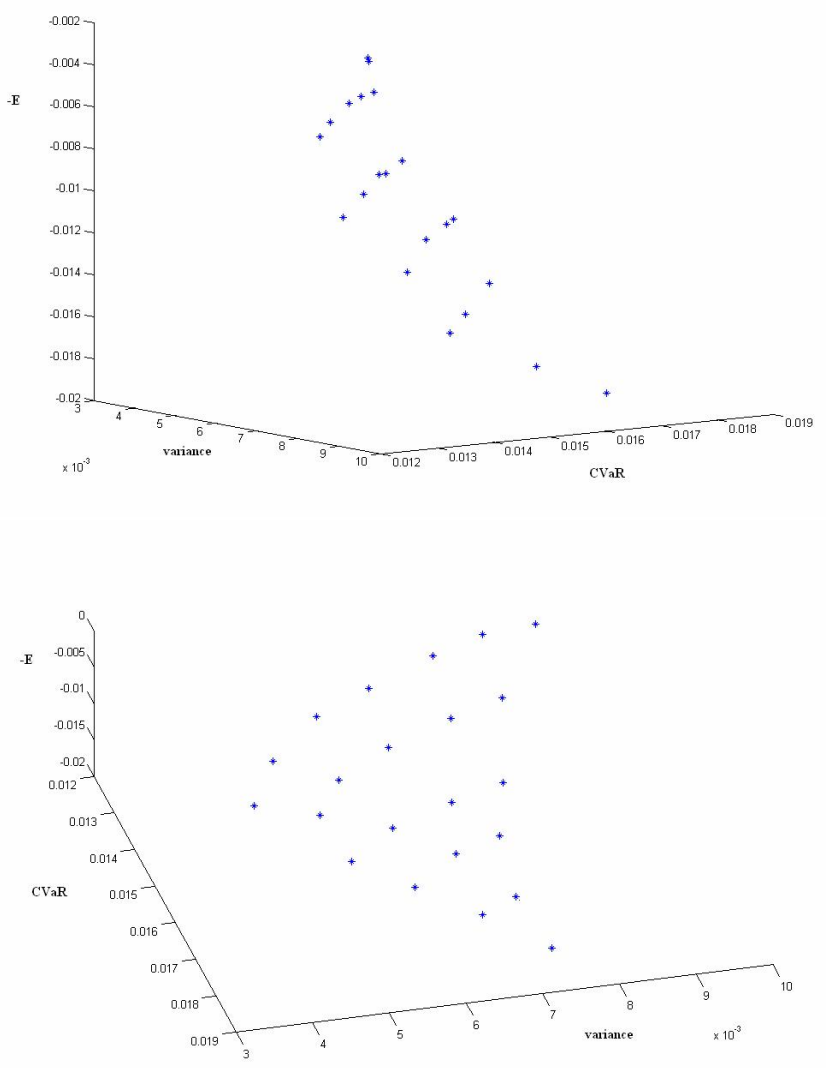

Figure 2: The result of NBI approach based on the hybrid method.

[3] I. Das, J. E. Dennis. Normal boundary intersection, a new methode for generating the Pareto surface in nonlinear multicreteria optimization problems. SIAM J. Optimization, 8 (1998), No. 3, 631-657.

[4] C. Bonnemoy. La méthode du recuit simulé: restauration des images, reconnaissance de Surfaces. R.A.I.R.O. Automatique-Productique Informatique Industrielle, 25 (1991), No. 5, 497-517.

[5] H. M. Markowitz. Portfolio selection. Journal of Finance, 1 (1952), No. 7, 77-91.

[6] J. C. Spall. Multivariate stochastic approximation using a simultaneous perturbation gradient approximation. IEEE Transactions on Automatic Control, 37 (1992), No. 3, 332-341.

[7] R. T. Rockafeller, S. Uryasev. Conditional value-at-risk for general loss distributions. Journal of Banking and Finance, 26 (2002), No. 7, 1443-1471. 
[8] Q. Yuan, Z. He, H. Leng. A hybrid genetic algorithm for a class of global optimization problems with box constraints. Applied Mathematics and Computation, 197 (2008), No. 2, 924929.

[9] I. G. Tsoulos. Modifications of real code genetic algorithm for global optimization. Applied Mathematics and Computation, 203 (2008), No. 2, 598-607. 